\title{
THE RECIPROCAL OF AN ENTIRE FUNCTION OF INFINITE ORDER AND THE DISTRIBUTION OF THE ZEROS OF ITS SECOND DERIVATIVE ${ }^{1}$
}

BY

JOHN ROSSI

\begin{abstract}
Let $f$ be a real entire function of infinite order whose zeros together with those of $f^{\prime}$ are all real. It is proved that $(1 / f)^{\prime \prime}$ has an infinity of nonreal zeros. The location of the zeros of $f^{\prime \prime}$ and $(1 / f)^{\prime \prime}$ is also investigated. The result complements a finite order result of Hellerstein and Williamson.
\end{abstract}

Introduction. A long open question of Pólya [8, 9] asks whether an entire function $f$ which has, along with each of its derivatives, only real zeros must have one of the following forms

$$
\begin{aligned}
& f(z)=A e^{B z} \\
& f(z)=A\left(e^{i c z}-e^{i d}\right)
\end{aligned}
$$

or

$$
f(z)=A z^{m} e^{-a z^{2}+b z} \prod_{n}\left(1-\frac{z}{a_{n}}\right) e^{z / a_{n}}
$$

where $A, B$ are complex constants, $a \geqslant 0, b, c, d$ and the $a_{n}$ are real, $m$ is a nonnegative integer and $\Sigma_{n} a_{n}^{-2}<\infty$. Functions of the form (1.3) are said to belong to the Laguerre-Pólya class.

For $f$ of finite order, the question was answered affirmatively by Hellerstein and Williamson [3] who needed only to assume that $f, f^{\prime}$, and $f^{\prime \prime}$ had only real zeros. For $f$ real (i.e. $z$ real implies $f(z)$ real) and of infinite order with $f$ and $f^{\prime}$ having only real zeros, an affirmative answer was obtained by Levin and Ostrovskiī [7] and Hellerstein and Williamson $[4]^{2}$ when they proved that $f^{\prime \prime}$ must have infinitely many nonreal zeros. Williamson has informed me that he, Hellerstein and L. C. Shen have recently found an affirmative answer to Pólya's question in the last remaining case when $f$ is nonreal and of infinite order.

Received by the editors August 12, 1980 and, in revised form, March 16, 1981. Presented to the Society at the annual meeting in San Francisco, January 9, 1981.

1980 Mathematics Subject Classification. Primary 30D35; Secondary 30D15.

Key words and phrases. Real entire function, real meromorphic function, infinite order.

${ }^{1}$ This paper is part of the author's Ph.D. thesis written under the direction of Jack Williamson and submitted to the University of Hawaii.

${ }^{2}$ The statement of Lemma 1 in [4] should read: The functions $\phi$ and $\phi_{1}$ are of order, at most, one and $\phi$ is in the Laguerre-Pólya class. Though the assertion in [4] that $\phi_{1}$ is also in the Laguerre-Pólya class is incorrect, it is never used in the sequel. 
In their proof of Pólya's conjecture for $f$ real and of finite order, Hellerstein and Williamson found a specific relationship between the growth of $f$ and the number of nonreal zeros of $f^{\prime \prime}$. To be precise we make the following definition.

For each integer $p \geqslant 0$, denote by $V_{2 p}$ the class of entire functions of the form

$$
f(z)=\exp \left(-a z^{2 p+2}\right) g(z)
$$

where $a \geqslant 0$ and $g(z)$ is a constant multiple of a real entire function of genus $\leqslant 2 p+1$ with only real zeros. That is, $g$ is of the form

$$
g(z)=c z^{m} e^{Q(z)} \prod_{n}\left(1-\frac{z}{z_{n}}\right) \exp \left[\frac{z}{z_{n}}+\cdots+\frac{1}{q}\left(\frac{z}{z_{n}}\right)^{q}\right]
$$

where $c$ is a constant, $m$ is a nonnegative integer, $Q$ is a real polynomial of degree $\leqslant 2 p+1, z_{n}$ is real for all $n, \Sigma_{p}\left|z_{n}\right|^{-q-1}<\infty$, and $q \leqslant 2 p+1$. Now set $U_{0}=V_{0}$ and for $p \geqslant 1, U_{2 p}=V_{2 p}-V_{2 p-2}$. We note that $U_{0}$ is the Laguerre-Pólya class of functions.

It was proved in [3] that if $f \in U_{2 p}$ and $f^{\prime}$ has only real zeros, then $f^{\prime \prime}$ has exactly $2 p$ nonreal zeros. Recently, Hellerstein and Williamson [5] found a remarkable "duality" between the nonreal zeros of $f$ " and the real zeros of $(1 / f)^{\prime \prime}$.

THEOREM A. If $f \in U_{2 p}$ and $f^{\prime}$ has only real zeros, then both the number of nonreal zeros of $f^{\prime \prime}$ and the number of real zeros of $(1 / f)^{\prime \prime}$ are equal to $2 p$.

They also showed

THEOREM B. Let $F=1 / f$ where $f$ is real entire and of finite order with $f$ and $f^{\prime}$ having only real zeros. Then

(a) $F^{\prime \prime}$ has only real zeros if and only if $f(z)=\exp \left(a z^{2}+b z+c\right), a \geqslant 0$, or $f(z)=(A z+B)^{n}, A \neq 0$, for some positive integer $n$.

(b) If $f$ has infinitely many (real) zeros, then $F^{\prime \prime}$ has infinitely many nonreal zeros.

By the assumption on the number of zeros of $f$ in Theorem $\mathrm{B}(\mathrm{b})$, it follows from Rolle's Theorem that $f^{\prime \prime}$ has infinitely many real zeros. So for $f$ of finite order, with infinitely many (real) zeros both the number of real zeros of $f^{\prime \prime}$ and the number of nonreal zeros of $(1 / f)^{\prime \prime}$ are infinite. Seen in this light, Theorem $\mathrm{B}(\mathrm{b})$ can be considered another "duality" result.

To understand the importance of Theorem B(a), one must understand Pólya's notion of final set for a meromorphic function $g$.

The final set $S(g)$ of $g$ is the set of all limit points of all the zeros of all the functions $g, g^{\prime}, g^{\prime \prime}, \ldots, g^{(n)}, \ldots$ It is understood that $z_{0} \in S(g)$ if $g^{(n)}\left(z_{0}\right)=0$ for infinitely many values of $n$.

Pólya has described the final sets for meromorphic functions in [10]. When applied to meromorphic functions $g$ with only real poles, his result can be stated as follows:

The final set $S(g)$ of $g$ is the set of perpendicular bisectors of the segments joining (distinct) consecutive poles. 
This says that for a meromorphic function $g$ with only real poles and at least two distinct ones, $g^{(n)}$ must eventually have nonreal zeros for $n$ large enough.

This fact together with the solution of Pólya's question for entire functions prompted Hellerstein and Williamson to pose the following:

PROBLEM. Characterize all meromorphic functions $F$ with only real poles such that $F$, $F^{\prime}$ and $F^{\prime \prime}$ have only real zeros.

Theorem $\mathrm{B}(\mathrm{a})$ solves this problem for a real meromorphic function of the form $F=1 / f$ where $f$ has finite order and shows that if $F$ has at least two distinct poles then $F^{\prime \prime}$ will have nonreal zeros. Thus already by the second derivative the zeros are beginning to approach Pólya's final set.

This may lead one to hope that all solutions to Hellerstein and Williamson's problem have at most one distinct pole. Hellerstein's simple counterexample $F(z)=$ $\tan z$ shows that this is not the case. Some indication that $\tan z$ and its translates may be the only counterexamples is investigated in Theorem 4.

It seems reasonable to ask whether Theorems $\mathrm{A}$ and $\mathrm{B}$ extend to functions of infinite order. The following theorem shows that the restriction of finite order may be removed from Theorem $B$.

THEOREM 1. Let $f$ be a (constant multiple of a) real entire function of infinite order with $f$ and $f^{\prime}$ having only real zeros. Then $(1 / f)^{\prime \prime}$ has infinitely many nonreal zeros.

As mentioned before the assumptions of Theorem 1 imply that $f^{\prime \prime}$ has infinitely many nonreal zeros. Thus in conjunction with Theorem A, we may ask whether $(1 / f)^{\prime \prime}$ has infinitely many real zeros. By taking $f(z)=\exp \left(e^{z}\right)$, we see that the answer is indeed no. Hellerstein, in written communication, asked whether the answer might be yes if it is further assumed that $f$ has infinitely many zeros. The following theorem shows that again the answer is no.

THEOREM 2. There exists a real entire function $f$ of infinite order with $f$ and $f^{\prime}$ having only real zeros such that $f$ has infinitely many zeros but $(1 / f)^{\prime \prime}$ has no real zeros.

In proving Theorem 2, two examples will be constructed, one of "high" infinite order, that is,

$$
\log T(r, f) \neq O(r \log r) \quad(r \rightarrow \infty)
$$

and one of "low" infinite order, that is,

$$
\log T(r, f)=O(r \log r) \quad(r \rightarrow \infty) .
$$

Here and throughout this paper, we use the standard symbols of Nevanlinna theory: $T(r, f), m(r, f)$, and $N(r, f)$. Also we will denote by $\lambda(f)$ the order of a meromorphic function $f$,

$$
\lambda(f)=\varlimsup_{r \rightarrow \infty} \frac{\log T(r, f)}{\log r} .
$$

The next results show where the zeros of $f^{\prime \prime}$ and $(1 / f)^{\prime \prime}$ are located if $f$ is of infinite order and satisfies (1.5) and a slightly weaker version of the conclusion of Theorem 2. Roughly speaking, these results show that the behavior of $f(z)=\exp \left(e^{z}\right)$ is "nearly" archetypical. In order to state the results clearly we introduce the 
following notation. For $\varepsilon$ arbitrary, $0<\varepsilon<\pi / 2$, set

$$
\begin{aligned}
& D_{1}=\left\{r e^{i \theta}: r>0,|\theta| \leqslant \frac{\pi}{2}-\varepsilon\right\}, \\
& D_{2}=\left\{r e^{i \theta}: r>0, \frac{\pi}{2}+\varepsilon \leqslant \theta \leqslant \pi-\varepsilon\right\}, \\
& D_{3}=\left\{r e^{i \theta}: r>0, \pi+\varepsilon \leqslant \theta \leqslant \frac{3 \pi}{2}-\varepsilon\right\},
\end{aligned}
$$

and the complementary regions

$$
\begin{aligned}
& E_{1}=\left\{r e^{i \theta}: r>0,|\theta-\pi| \leqslant \frac{\pi}{2}-\varepsilon\right\}, \\
& E_{2}=\left\{r e^{i \theta}: r>0, \varepsilon \leqslant \theta \leqslant \frac{\pi}{2}-\varepsilon\right\}, \\
& E_{3}=\left\{r e^{i \theta}: r>0,-\frac{\pi}{2}+\varepsilon \leqslant \theta \leqslant-\varepsilon\right\} .
\end{aligned}
$$

Finally, set

$$
D=\bigcup_{k=1}^{3} D_{k} \text { and } E=\bigcup_{k=1}^{3} E_{k}
$$

We may now state

THEOREM 3. Let $f$ be a (constant multiple of a) real entire function of infinite order with $f$ and $f^{\prime}$ having only real zeros. Assume that $f$ satisfies $(1.5)$ and that $(1 / f)^{\prime \prime}$ has only finitely many real zeros. Then either $D$ or $E$ contains only finitely many zeros of $f^{\prime \prime}$ and $(1 / f)^{\prime \prime}$.

COROllary 3.1. Let $f$ be as in Theorem 3. Furthermore, assume that $f^{\prime}$ has only finitely many zeros. Then only finitely many zeros of $f^{\prime \prime}$ and $(1 / f)^{\prime \prime}$ lie in $D_{1} \cup E_{1}$.

(It should be noted in Corollary 3.1 that the assumption on the reality of the zeros of $f$ and the finite number of zeros of $f^{\prime}$ together with Rolle's theorem imply that $f$ has finitely many zeros as well.)

It is shown in [4] that if $f$ is real entire with only real zeros and satisfies (1.5), then

$$
\frac{f^{\prime}}{f}(z)=\phi(z) \psi(z)
$$

where $\phi$ is real entire, $\lambda(\phi) \leqslant 1$, and $\psi$ is real meromorphic and maps $\operatorname{Im} z>0$ to the upper half plane. Then since $f^{\prime}$ is assumed to have only real zeros, $f^{\prime \prime} / f^{\prime}$ may be written as

$$
\frac{f^{\prime \prime}}{f^{\prime}}(z)=\phi_{1}(z) \psi_{1}(z)
$$

where $\phi_{1}$ and $\psi_{1}$ correspond to $\phi$ and $\psi$ in (1.9). If in addition we assume as in Corollary 3.1 that $f^{\prime}$ has only finitely many (real) zeros, then by construction $\psi_{1}$ is rational. We may now state

COROllary 3.2. Let $f$ be as in Corollary 3.1. Furthermore, assume that $f^{\prime}$ has at least one zero. Then given $a \in \mathbf{C}$, there exist only finitely many solutions to $\phi_{1}(z)=a$ in $D_{1} \cup E_{1}$. 
Our final result concerns functions mapping $\operatorname{Im} z>0$ to the upper half plane.

THEOREM 4. Let $\psi$ be any real meromorphic function mapping $\operatorname{Im} z>0$ to the upper half plane. If $\psi^{\prime}$ and $\psi^{\prime \prime}$ have only real zeros, then $\lambda(\psi) \leqslant 1$.

Theorem 4 is of interest since Hellerstein's counterexample $F(z)=\tan z$ maps $\operatorname{Im} z>0$ to the upper half plane. Theorem 4 says that if $\psi, \psi^{\prime}, \psi^{\prime \prime}$ have only real zeros and $\psi$ maps $\operatorname{Im} z>0$ to the upper half plane, then $\psi$ "grows" like $\tan z$.

I would like to thank Professors Simon Hellerstein and Jack Williamson for suggesting the problem solved in Theorem 1 and for bringing to my attention Pólya's notion of final set.

2. Preliminaries. The following theorem of Levin and Ostrovskiı̌ [7] allows us to limit our attention to functions of infinite order satisfying (1.5).

THEOREM C. If all the zeros of $f(z)$ are real and the zeros $\left\{a_{k}\right\}_{k}$ of either $f^{\prime \prime}(z)$ or $(1 / f)^{\prime \prime}(z)$ are such that $\Sigma_{k}\left|\operatorname{Im}\left(a_{k}^{-1}\right)\right|<+\infty$, then (1.5) holds.

(It should be pointed out that in [7] this theorem is stated with no mention of $(1 / f)^{\prime \prime}$. However, by the remark preceding $\$ 3$ in [7], we see that their Theorem 1 holds for $1 / f$ as well as for $f$. Since

$$
\frac{(1 / f)^{\prime}}{(1 / f)}=-\frac{f^{\prime}}{f},
$$

the conclusion in their Theorem 2 is still valid for $f$.)

By Theorem C, we see that in our Theorem 1, (1.5) may be assumed without loss of generality. Therefore for the rest of this paper unless otherwise stated $f$ will represent a function satisfying the hypothesis of Theorem 1 and (1.5). In seeking a representation for $f^{\prime} / f$, denote by $\left\{a_{k}\right\}_{k}$ the distinct zeros of $f$ and enumerate them as follows:

$$
\cdots<a_{k-1}<a_{k}<a_{k+1}<\cdots \quad(-\infty \leqslant \alpha \leqslant k \leqslant \omega \leqslant+\infty, k \text { finite }) .
$$

By Rolle's theorem, $f^{\prime}$ has at least one zero in each interval $\left(a_{k}, a_{k+1}\right)$. Choose exactly one zero and denote it by $b_{k}$. Then

$$
a_{k}<b_{k}<a_{k+1} \text { for all } k \text {. }
$$

Also order the $a_{k}$ 's and $b_{k}$ 's in such a way that

$$
b_{-1}<0<a_{1} \text {. }
$$

Now set

$$
\psi(z)= \begin{cases}\frac{z-b_{0}}{z-a_{0}} \prod_{k \neq 0}\left(\frac{1-z / b_{k}}{1-z / a_{k}}\right), & \text { if } \omega=+\infty, \\ \frac{z-b_{0}}{\left(z-a_{0}\right)\left(a_{\omega}-z\right)} \prod_{k \neq 0}\left(\frac{\left(1-z / b_{k}\right)}{\left(1-z / a_{k}\right)}\right), & \text { if } \omega<+\infty .\end{cases}
$$

If $f$ has no zeros, set $\psi(z) \equiv 1$, if it has only one distinct zero $a_{1}$, set $\psi(z)=$ $\left(a_{1}-z\right)^{-1}$, and if $f$ has only two distinct zeros $a_{1}, a_{2}\left(a_{1}<a_{2}\right)$, choose a zero $b_{1}$ of 
$f^{\prime}$ in $\left(a_{1}, a_{2}\right)$ and set

$$
\psi(z)=\frac{\left(z-b_{1}\right)}{\left(z-a_{1}\right)\left(a_{2}-z\right)} .
$$

We have the following lemma [6, pp. 308-309].

Lemma 1. Let $\psi(z)$ be a real meromorphic function. Then $\psi(z)$ maps $\operatorname{Im} z>0$ to the upper half plane if and only if

$$
\psi(z)=c \psi_{1}(z) L(z)
$$

where $\psi_{1}$ is of the form (2.4),c>0 and $L(z)$ has one of the following forms

$$
1, z-b_{\alpha^{\prime}}, b_{\omega}-z,\left(z-b_{\alpha^{\prime}}\right)\left(b_{\omega}-z\right) \quad\left(b_{\alpha^{\prime}}<a_{\alpha}, b_{\omega}<\alpha_{\omega}\right) .
$$

Hellerstein and Williamson show in [4] that

$$
\left(f^{\prime} / f\right)(z)=\phi(z) \psi(z)
$$

where $\lambda(\phi) \leqslant 1$ and $\psi$ has the form (2.4).

The following lemma, whose proof may be found in [6, pp. 310-311] gives an alternate representation of $\psi(z)$.

LeMma 2. Let $\psi(z)$ be real meromorphic. If $\psi(z)$ maps $\operatorname{Im} z>0$ to the upper half plane $\psi(z)$ may be written

$$
\psi(z)=\gamma z+\delta+\sum_{k=\alpha}^{\omega} A_{k}\left(\frac{1}{a_{k}-z}-\frac{1}{a_{k}}\right)
$$

where $A_{k}>0, \gamma \geqslant 0, \delta$ is real and $\sum_{k=\alpha}^{\omega} A_{k} / a_{k}^{2}<+\infty$.

We now state three technical lemmas concerning the growth of $\psi(z)$. A sketch of the proof of Lemma 3 may be found in [5]. The proof of Lemmas 4 and 5 use standard techniques and may be found in [11].

Lemma 3. For all $z \in \mathbf{C}-\mathbf{R}$, we have

$$
\frac{\left|\psi^{\prime}(z)\right|}{|\operatorname{Im} \psi(z)|} \leqslant \frac{1}{|\operatorname{Im} z|} \text {. }
$$

Note that Lemma 3 implies that for $0<\varepsilon<\pi / 2$,

$$
\left|\frac{\psi^{\prime}}{\psi}(z)\right| \rightarrow 0
$$

uniformly as $|z| \rightarrow \infty, \varepsilon \leqslant|\arg z| \leqslant \pi-\varepsilon$. The next lemma improves this result for functions $\psi$ with only finitely many zeros and poles on the positive axis.

LEMMA 4. Let $\psi$ have finitely many zeros and poles on the positive real axis, then for $0<\varepsilon<\pi / 2$,

$$
\left|\frac{\psi^{\prime}}{\psi}(z)\right| \rightarrow 0
$$

uniformly as $|z| \rightarrow \infty,|\arg z| \leqslant \pi-\varepsilon$. 
The Carathéodory estimate for functions mapping $\operatorname{Im} z>0$ to the upper half plane [6, pp. 18-19] states that there exist positive constants $c_{1}, c_{2}$ depending on $\psi$ such that

$$
c_{2}|\sin \theta| r^{-1} \leqslant|\psi(z)| \leqslant c_{1}|\sin \theta|^{-1} r \quad(r \geqslant 1, \theta \neq 0, \pi) .
$$

The next lemma improves (2.10) for functions of the form (2.4) with $\omega<+\infty$.

LEMMA 5. Let $\psi$ be of the form (2.4) with $\omega<+\infty$. Then for $0<\varepsilon<\pi / 2$,

$$
|\psi(z)|=O(1) \quad(|z| \rightarrow \infty,|\arg z| \leqslant \pi-\varepsilon) .
$$

It should be noted that the obvious analogues of Lemmas 4 and 5 hold if $\psi$ has only finitely many zeros and poles on the negative axis instead of the positive axis.

The next lemma is a kind of converse to Lemma 2.

LEMMA 6. Let $\psi(z)$ have the form

$$
\psi(z)=\sum_{k=1}^{\infty} A_{k}\left(\frac{1}{a_{k}-z}-\frac{1}{a_{k}}\right)
$$

where all the $a_{k}$ 's are distinct and positive with $a_{k}<a_{k+1}, \lim _{k \rightarrow \infty} a_{k}=+\infty$ and $A_{k}>0$ for all $k$. Furthermore, suppose

$$
\sum_{k=1}^{\infty} \frac{A_{k}}{a_{k}}<\infty
$$

Then $\psi(z)$ is meromorphic in $\mathbf{C}$ with simple poles at the $a_{k}$ and maps $\operatorname{Im} z>0$ to the upper half plane.

Proof. The proof that $\psi$ is meromorphic is standard and will be left to the reader. To see that $\psi(z)$ maps $\operatorname{Im} z>0$ to the upper half plane simply note that

$$
\begin{aligned}
\operatorname{Im}(\psi(z)) & =\sum_{k=1}^{\infty} A_{k} \operatorname{Im}\left(\frac{1}{a_{k}-z}\right) \\
& =\sum_{k=1}^{\infty} A_{k} \operatorname{Im}\left(\frac{a_{k}-\bar{z}}{\left|a_{k}-z\right|^{2}}\right)=(\operatorname{Im} z) \sum_{k=1}^{\infty} \frac{A_{k}}{\left|a_{k}-z\right|^{2}} .
\end{aligned}
$$

Since $A_{k}>0$ for all $k$, the result is obvious and the proof of Lemma 6 is complete.

The last two growth lemmas are needed in the proof of Theorem 1. The proof of Lemma 7 is an easy growth argument while a sketch of the proof of Lemma 8 may be found in [5].

LEMMA 7. Let $\Pi(z)$ be a canonical product of genus $p$ with only real zeros. Let $\varepsilon>0$ and let

$$
G=\left\{z=r e^{i \theta}: r>0, \varepsilon \leqslant|\theta| \leqslant \pi-\varepsilon\right\}
$$

Then

$$
\left|\frac{\Pi^{\prime}}{\Pi}(z)\right|=o\left(r^{p}\right) \quad(|z|=r \rightarrow \infty, z \in G) .
$$


LEMMA 8. Let $g(z)$ be a real entire function of genus 0 or 1 with only real zeros. If $g(z)$ has infinitely many zeros, then for $y$ real and for any positive integer $n$

$$
\lim _{\lfloor y \mid \rightarrow \infty}|g(i y)||y|^{-n}=\infty \text {. }
$$

3. Proof of Theorem 1. The proof of Theorem 1 depends heavily on the following theorem due to Edrei [1].

THEOREM D. Let $h(z)$ be meromorphic with all but finitely many of the roots of

$$
\begin{gathered}
h(z)=0, \\
h(z)=\infty, \\
h^{(n)}(z)=1 \quad\left(n \geqslant 0, h^{(0)} \equiv h\right)
\end{gathered}
$$

lying on the radii

$$
r e^{i \omega_{1}}, r e^{i \omega_{2}}, \ldots, r e^{i \omega_{4}} \quad(r>0)
$$

where $0 \leqslant \omega_{1}<\omega_{2}<\cdots<\omega_{q}<2 \pi(q \geqslant 1)$.

Assume that the roots of one of the equations (3.1), (3.2), (3.3) has a finite exponent of convergence $\rho$. Define

$$
\beta=\sup \left\{\frac{\pi}{\omega_{2}-\omega_{1}}, \frac{\pi}{\omega_{3}-\omega_{2}}, \ldots, \frac{\pi}{\omega_{q+1}-\omega_{q}}\right\}
$$

where $\omega_{q+1}=2 \pi+\omega_{1}$. Then $\lambda(h)$ is finite and either

$$
\rho=\lambda \text { or } \rho<\lambda \leqslant \beta \text {. }
$$

Two additional lemmas are needed for the proof of Theorem 1.

Set $F=1 / f$ and recall the assumption of $f$ stated at the beginning of $\$ 2$.

LEMMA 9. If $F^{\prime \prime}$ has only finitely many nonreal zeros, then it has infinitely many real zeros.

Proof. Assume that

$$
F^{\prime \prime} \text { has only finitely many real zeros. }
$$

By (2.6) and the fact that $f^{\prime}$ has only real zeros we have

$$
f^{\prime} / f=\phi \psi
$$

where $\phi$ is in the Laguerre-Pólya class, $\lambda(\phi) \leqslant 1$, and $\psi$ is of the form (2.4).

Since

$$
F^{\prime} / F=-f^{\prime} / f
$$

Rolle's theorem, (3.7)-(3.9) and the interlacing of the $a_{k}$ 's and $b_{k}$ 's ((2.1) and (2.2)) imply that $\phi$ has finitely many zeros. Thus,

$$
\phi(z)=e^{\alpha z} P(z)
$$

where $\alpha$ is a real constant and $P(z)$ is a polynomial with only real zeros. By an argument in [4, pp. 500-502], it is seen that since $f$ has infinite order

$$
\alpha \neq 0 \text {. }
$$


We assume that

$$
\alpha<0 \text {. }
$$

The argument when $\alpha>0$ is analogous. The argument mentioned in [4] shows that $f$ and $f^{\prime}$ have only finitely many zeros off the negative real axis.

Assume for the moment that

$$
\lambda\left(f^{\prime} / f\right) \leqslant \frac{1}{2} .
$$

Once (3.13) is established, the lemma is proved as follows, using the techniques of Nevanlinna theory. By (3.8) and (3.10)

$$
T\left(r, e^{\alpha z}\right)=m\left(r, e^{\alpha z}\right) \leqslant m\left(r, \frac{1}{P}\right)+m\left(r, \frac{1}{\psi}\right)+m\left(r, \frac{f^{\prime}}{f}\right) .
$$

By the Caratheodory estimate (2.10) on $\psi$ we have $m(r, 1 / \psi)=O(\log r)(r \rightarrow \infty)$. This together with the rationality of $1 / P$ and (3.14) give

$$
T\left(r, e^{\alpha z}\right) \leqslant m\left(r, f^{\prime} / f\right)+O(\log r) \quad(r \rightarrow \infty) .
$$

But by (3.13), this means $\lambda\left(e^{\alpha z}\right) \leqslant 1 / 2$. This is a contradiction since $\alpha \neq 0$. Hence (3.7) is false and the lemma true.

Thus it remains to verify (3.13). To do this, note first by (3.8)-(3.10) that all but finitely many roots of the equations

$$
\begin{aligned}
& F / F^{\prime}=0, \\
& F / F^{\prime}=\infty,
\end{aligned}
$$

lie on the negative real axis. Furthermore,

$$
\left(\frac{F}{F^{\prime}}\right)^{\prime}=1 \Leftrightarrow\left(\frac{F}{F^{\prime}}\right)\left(\frac{F^{\prime \prime}}{F^{\prime}}\right)=0 \Leftrightarrow\left(\frac{f}{f^{\prime}}\right)\left(\frac{F^{\prime \prime}}{F^{\prime}}\right)=0 .
$$

Now

$$
\frac{f}{f^{\prime}}=0 \Rightarrow\left(\frac{f}{f^{\prime}}\right)\left(\frac{F^{\prime \prime}}{F^{\prime}}\right) \neq 0
$$

To see this suppose $z_{0}$ is a zero of $f$ of multiplicity $m$. Then $z_{0}$ is a pole of $F^{\prime}$ of multiplicity $m+1$ and therefore a pole of $F^{\prime \prime} / F^{\prime}$ of multiplicity one. Thus $\left(f / f^{\prime}\right)\left(z_{0}\right) \cdot\left(F^{\prime \prime} / F^{\prime}\right)\left(z_{0}\right) \neq 0$ and (3.19) is true. Therefore (3.18) and (3.19) imply

$$
\left(\frac{F}{F^{\prime}}\right)^{\prime}=1 \Leftrightarrow\left(\frac{F^{\prime \prime}}{F^{\prime}}\right)=0 \text {. }
$$

Thus by hypothesis and assumption (3.7), we have that the equation

$$
\left(F / F^{\prime}\right)^{\prime}=1
$$

has only finitely many roots. By (3.16), (3.17) and (3.21), we may use Theorem D with $\rho=0$ for (3.21) and $\beta=\frac{1}{2}$ and obtain $\lambda\left(F / F^{\prime}\right) \leqslant \frac{1}{2}$. But since $\lambda\left(F / F^{\prime}\right)=$ $\lambda\left(f^{\prime} / f\right),(3.13)$ is true and the proof of Lemma 9 is complete.

LEMMa 10. Assume $F^{\prime \prime}$ has only finitely many nonreal zeros. Then

$$
\lambda\left(f^{\prime} / f\right) \leqslant 1 \text {. }
$$


Proof. We have that all but finitely many of the roots of (3.16), (3.17), and (3.21) lie on the real axis. Then in the notation of Theorem $D, \beta=1$. We need only show that the exponent of convergence $\rho$ for the roots of equation (3.21) satisfies $\rho \leqslant 1$.

To do this we use an argument found in [5]. (See the authors' summary after (3.35).) Let $\left\{\beta_{n}\right\}_{n}$ be the distinct zeros of $f^{\prime} / f$ enumerated in the same way as in (2.1). By (2.4) and (2.6) we see that this sequence is made up of the $b_{k}$ 's and the distinct zeros of $\phi(z)$ (which are real since $f^{\prime}$ has only real zeros) not contained in $\left\{b_{k}\right\}$. We call any interval $\left(\beta_{n}, \beta_{n+1},\right)$ which contains no zero of $f$ an atypical interval of the zeros of $f^{\prime} / f$. If $f^{\prime} / f$ has a least zero $\beta_{\alpha}$ and the interval $\left(-\infty, \beta_{\alpha}\right)$ contains no zero of $f$ then $\left(-\infty, \beta_{\alpha}\right)$ is called a semi-infinite atypical interval. A similar definition applies when $f^{\prime} / f$ has a greatest zero.

For $f$ of finite order, Hellerstein and Williamson in [5] were able to locate all the real zeros of $F^{\prime \prime}$. Clearly real zeros of $F^{\prime \prime}$ arise from multiple zeros of $f^{\prime} / f$. The only other real zeros of $F^{\prime \prime}$ occur in atypical intervals of the zeros of $f^{\prime} / f$, exactly one in each finite atypical interval and at most one in each semi-infinite atypical interval. This result depended on the fact that $\phi$ was a polynomial satisfying

$$
\left.\left(\phi^{\prime} / \phi\right)^{\prime} \leqslant 0 \quad \text { (for } x \text { real, } \phi(x) \neq 0\right) \text {. }
$$

In the case considered here $\phi$ is in the Laguerre-Pólya class and thus has the form (1.3). An easy verification shows that any such function satisfies (3.23) as well; hence, the result in [5] applies here.

Bearing in mind that multiple zeros of $F^{\prime} / F$ are zeros of $\phi$, these considerations show that $\rho\left(F^{\prime \prime} / F^{\prime}\right) \leqslant \rho(\phi)$ where $\rho\left(F^{\prime \prime} / F^{\prime}\right)$ and $\rho(\phi)$ denote the exponents of convergence of $F^{\prime \prime} / F^{\prime}$ and $\phi$ respectively. By $(2.6) \lambda(\phi) \leqslant 1$ and so $\rho\left(F^{\prime \prime} / F^{\prime}\right) \leqslant$ $\rho(\phi) \leqslant \lambda(\phi) \leqslant 1$. By (3.20) we have $\rho \leqslant 1$ and the lemma is proved.

Proof of TheOREM 1. Assume $F^{\prime \prime}$ has only finitely many nonreal zeros. Let $\left\{\gamma_{k}\right\}_{\alpha^{\prime}}^{\omega^{\prime}-1}$ be the subcollection of the distinct zeros of $F^{\prime}=\left(-f^{\prime} / f^{2}\right)$ such that $\gamma_{k}$ is the left-hand endpoint of a finite atypical interval ordered as in (2.1) with $k$ finite and $-\infty \leqslant \alpha^{\prime} \leqslant k \leqslant \omega^{\prime}-1 \leqslant+\infty$. If $\omega^{\prime}-1<+\infty$, we denote by $\gamma_{\omega^{\prime}}$ the righthand endpoint of the atypical interval $\left(\gamma_{\omega^{\prime}-1}, \gamma_{\omega^{\prime}}\right)$. By the argument in [5] just mentioned, it is easy to see that there is exactly one zero $\delta_{k}$ of $F^{\prime \prime}$ in each interval $\left(\gamma_{k}, \gamma_{k+1}\right)$. Construct $\psi_{0}(z)$ in exactly the same way $\psi(z)$ was constructed in (2.4) with $a_{k}$ replaced by $\gamma_{k}, b_{k}$ by $\delta_{k}, \alpha$ by $\alpha^{\prime}$ and $\omega$ by $\omega^{\prime}$. Clearly by Lemma $1, \psi_{0}$ maps $\operatorname{Im} z>0$ to the upper half plane.

The aforementioned argument in [5] together with (3.23) shows that the $\delta_{k}$ account for all but possibly two of the real zeros of $F^{\prime \prime} / F^{\prime}$. Then since $F^{\prime \prime}$ is assumed to have only finitely many nonreal zeros, $F^{\prime \prime} / F^{\prime}$ may be written in the form

$$
\frac{F^{\prime \prime}}{F^{\prime}}=\frac{P \psi_{0}}{g}
$$

where $P$ is a polynomial containing the finitely many nonreal zeros of $F^{\prime \prime}$ and the two possible zeros of $F^{\prime \prime}$ in the semi-infinite atypical intervals, $g$ is real entire with only real zeros and $\psi_{0}$ maps $\operatorname{Im} z>0$ to the upper half plane and is of the form (2.4). We will now show that our assumption on the number of nonreal zeros of $F^{\prime \prime}$ and representation (3.24) lead to a contradiction. 
First, we show that

$$
\lambda(g) \leqslant 1 \text {. }
$$

To see this we note that by (3.24) and the standard estimates of Nevanlinna theory,

$$
m(r, g) \leqslant m(r, P)+m\left(r, \psi_{0}\right)+T\left(r, \frac{F^{\prime \prime}}{F^{\prime}}\right)+O(1) \quad(r \rightarrow \infty) .
$$

The first two terms on the right-hand side of (3.26) are $O(\log r)$; the first since $P$ is a polynomial, the second since $\psi_{0}$ satisfies the Carathéodory estimate (2.10). Now

$$
T\left(r, \frac{F^{\prime \prime}}{F^{\prime}}\right)=m\left(r, \frac{F^{\prime \prime}}{F^{\prime}}\right)+N\left(r, \frac{F^{\prime \prime}}{F^{\prime}}\right) .
$$

Nevanlinna's lemma on the logarithmic derivative [2, p. 36] and (1.5) imply

$$
m\left(r, \frac{F^{\prime \prime}}{F^{\prime}}\right)=O(r \log r) \quad(r \rightarrow \infty) .
$$

Since

$$
\begin{aligned}
\frac{F^{\prime \prime}}{F^{\prime}} & =\frac{\left(f^{\prime} / f\right)^{\prime}}{\left(f^{\prime} / f\right)}-\frac{f^{\prime}}{f}, \\
N\left(r, \frac{F^{\prime \prime}}{F^{\prime}}\right) & \leqslant N\left(r, \frac{\left(f^{\prime} / f\right)^{\prime}}{\left(f^{\prime} / f\right)}\right)+N\left(r, \frac{f^{\prime}}{f}\right) \\
& \leqslant 2 N\left(r, \frac{f^{\prime}}{f}\right)+N\left(r, \frac{f}{f^{\prime}}\right) \\
& \leqslant 3 T\left(r, \frac{f^{\prime}}{f}\right)+O(1) \quad(r \rightarrow \infty) .
\end{aligned}
$$

Thus (3.26)-(3.30) imply

$$
m(r, g) \leqslant O(r \log r)+3 T\left(r, f^{\prime} / f\right) .
$$

Lemma 10 and (3.31) now yield (3.25).

Notice that on the imaginary axis

$$
|g(i y)| \geqslant k_{1}>0 \quad\left(|y| \geqslant y_{0}, k_{1}>0 \text { constant }\right) .
$$

This is clear if $g$ has infinitely many zeros since by (3.25) and the fact that $g$ has only real zeros, Lemma 8 applies. If $g$ has finitely many zeros, then again by (3.25) and the fact that $g$ is real entire

$$
g(z)=e^{\alpha z} P_{1}(z)
$$

where $\alpha$ is real and $P_{1}$ is a polynomial $\left(P_{1} \neq 0\right.$ ). Clearly (3.32) holds in this case.

Furthermore by the Carathéodory estimate (2.10) for $\psi_{0}$ we have

$$
\left|\psi_{0}(i y)\right| \leqslant k_{2}|y| \quad\left(k_{2}>0, \text { constant }\right) .
$$

Thus (3.24), (3.32) and (3.33) give

$$
\left|\frac{F^{\prime \prime}}{F^{\prime}}(i y)\right| \leqslant k_{3}|y|^{d+1} \quad\left(|y| \geqslant y_{0}>0\right)
$$

where $k_{3}$ is a positive constant and $d=\operatorname{degree} P$. 
On the other hand representations (3.29) and (3.8) give

$$
\begin{aligned}
\left|\frac{F^{\prime \prime}}{F^{\prime}}(i y)\right| & =\left|\frac{\phi^{\prime}}{\phi}(i y)+\frac{\psi^{\prime}}{\psi}(i y)-\phi(i y) \psi(i y)\right| \\
& \geqslant|\phi(i y)||\psi(i y)|-\left(\left|\frac{\phi^{\prime}}{\phi}(i y)\right|+\left|\frac{\psi^{\prime}}{\psi}(i y)\right|\right) .
\end{aligned}
$$

By (3.8), $\phi$ is in the Laguerre-Pólya class with $\lambda(\phi) \leqslant 1$. Thus

$$
\phi(z)=e^{\alpha z+\beta} \Pi(z)
$$

where $\alpha, \beta$ are real and $\Pi$ is a canonical product of genus 0 or 1 . Applying Lemma 7 to $\Pi$ with $p=0$ or 1 we obtain from (3.36)

$$
\left|\frac{\phi^{\prime}}{\phi}(i y)\right|=o(|y|) \quad(|y| \rightarrow \infty) .
$$

By Lemma 3

$$
\left|\frac{\psi^{\prime}}{\psi}(i y)\right|=o(1) \quad(|y| \rightarrow \infty)
$$

and by the Carathéodory estimate (2.10) for $\psi$

$$
|\psi(i y)| \geqslant k_{4}|y|^{-1} \quad\left(|y| \geqslant y_{0}, k_{4}>0 \text {, constant }\right) .
$$

Since we have assumed that $F^{\prime \prime}$ has only finitely many nonzero zeros, Lemma 9 implies that $F^{\prime \prime}$ has infinitely many real zeros. By the correspondence between the real zeros of $F^{\prime \prime}$ and the zeros of $\phi, \phi$ has infinitely many zeros as well. Since $\phi$ has genus $\leqslant 1$, Lemma 8 implies

$$
|\phi(i y)| \neq O\left(|y|^{n}\right) \quad(|y| \rightarrow \infty)
$$

for any positive integer $n$. Then (3.35)-(3.40) give

$$
\left|\frac{F^{\prime \prime}}{F^{\prime}}(i y)\right| \neq O\left(|y|^{n}\right) \quad(|y| \rightarrow \infty)
$$

for any positive integer $n$.

Clearly (3.41) and (3.34) are incompatible and thus the assumption that $F^{\prime \prime}$ had finitely many nonreal zeros is false. This completes the proof of Theorem 1 .

\section{Proof of Theorem 2.}

(a) Construction of $f$ with high infinite order. Define $f(z)$ as follows

$$
f(z)=h(\sin z)
$$

where

$$
h(z)=z \exp \left(\int_{\Gamma_{z}}\left(\frac{e^{\zeta}-1}{\zeta}\right) d \zeta\right)
$$

with $\Gamma_{z}$ any path from 0 to $z$.

$f(z)$ can then be written as

$$
f(z)=\sin z \exp \left(\int_{\Gamma_{\sin z}}\left(\frac{e^{\zeta}-1}{\zeta}\right) d \zeta\right) .
$$


Since $\left(e^{z}-1\right) / z$ is entire, so is $f(z)$. Furthermore, a computation gives

$$
\frac{f^{\prime}}{f}(z)=\exp (\sin z) \cot z
$$

Now $f$ has only simple, real zeros. Also (4.1) and (4.2) show that $f$ satisfies the hypotheses of Theorem 2. It is evident from (4.1) that $f(z)$ grows on the order of $\exp \left[\exp \left(e^{z}\right)\right]$ and thus $\log T(r, f) \neq O(r \log r)(r \rightarrow \infty)$.

If $F=1 / f$, another calculation gives

$$
\frac{F^{\prime \prime}}{F^{\prime}}(z)=\frac{\cos ^{2} z[\sin z-\exp (\sin z)]-1}{\sin z \cos z} .
$$

Since $\cos ^{2} x \geqslant 0$ and $\sin x \leqslant \exp (\sin x)(x=\operatorname{Re} z),(4.3)$ implies that $F^{\prime \prime} / F^{\prime}$ has no real zeros. Also, since

$$
\frac{F^{\prime}}{F}(z)=-\exp (\sin z) \cot z,
$$

all the zeros of $F^{\prime}$ are simple. Thus $F^{\prime \prime}$ has no real zeros.

It should be noted that Theorem 1 insures that $F^{\prime \prime}$ has infinitely many nonreal zeros.

(b) Construction of $f$ with low infinite order. First define

$$
h(z)=-\frac{e^{z} \psi(z)}{z}
$$

with

$$
\psi(z)=\sum_{n=1}^{\infty} \frac{\log a_{n}}{a_{n}}\left[\frac{1}{\left(\log a_{n}\right)-z}-\frac{1}{\log a_{n}}\right]
$$

where $a_{n}>1$ and $a_{n}<a_{n+1}$ for all $n, a_{n} \rightarrow \infty$ as $n \rightarrow \infty$,

$$
\sum_{n=1}^{\infty} \frac{1}{a_{n}}<+\infty
$$

and

$$
\sum_{n=1}^{\infty}\left(\log a_{n}\right)^{-\rho}=+\infty, \quad \text { for all } \rho>0 .
$$

(Note that the sequence $\left\{n^{2}+1\right\}_{1}^{\infty}$ satisfies all these conditions.)

Then $\psi(z)$ satisfies the hypothesis of Lemma 6 and

$$
\operatorname{Res}\left[h(z), \log a_{n}\right]=-\frac{\exp \left(\log a_{n}\right)}{\log a_{n}}\left[-\left(\frac{\log a_{n}}{a_{n}}\right)\right]=1 .
$$

Thus $h(z)$ is meromorphic with all residues equal to one. This means (see [12, p. 340]) that there exists an entire function $f(z)$ with simple zeros at $\left\{\log a_{n}\right\}_{1}^{\infty}$ such that

$$
\frac{f^{\prime}}{f}(z)=-\frac{e^{z}}{z} \psi(z)
$$

We notice that $\log a_{1}$ is the smallest pole of $\psi(z)$. Since $\psi(0)=0$ and $0<\log a_{1}$, Lemma 1 gives

$$
\psi(z)=c z \psi_{1}(z)
$$


where $c>0$ and $\psi_{1}(z)$ is of the form (2.4). Thus

$$
\left(f^{\prime} / f\right)(z)=-c e^{z} \psi_{1}(z) \text {. }
$$

Now $f^{\prime} / f$ is in the form (3.8) insuring that $f^{\prime}$ has only real zeros. Furthermore, $f$ is of infinite order. Indeed, by (4.7), its zeros have an infinite exponent of convergence.

By (4.9) $\phi(z)$ in (3.8) has the form

$$
\phi(z)=-c e^{z} .
$$

Thus by (3.8) and (4.10) $f^{\prime} / f$ has no multiple zeros and in the terminology of [5], $f^{\prime} / f$ has no atypical intervals. Hence, according to the summary after (3.35) in [5] mentioned before, $F^{\prime \prime}$ has no real zeros.

By (4.9) and an argument in [7, pp. 336-337], $\log T(r, f)=O(r \log r)(r \rightarrow \infty)$. The proof of Theorem 2 is now complete.

5. Proof of Theorem 3. The hypothesis of Theorem 3 implies that (3.7) holds and therefore (3.8), (3.10) and (3.11) hold. We assume throughout that

$$
\alpha<0
$$

and prove that only finitely many zeros of $f^{\prime \prime}$ and $(1 / f)^{\prime \prime}$ lie in $D$. An almost identical argument for $\alpha>0$ shows that only finitely many zeros of $f^{\prime \prime}$ and $(1 / f)^{\prime \prime}$ lie in $E$.

We first prove the statement regarding the zeros of $f^{\prime \prime}$. As was mentioned in the proof of Lemma 9, (3.8), (3.10) and (5.1) imply that $f$ and $f^{\prime}$ have only finitely many zeros off the negative axis. Thus (3.8)-(3.10) yield

$$
\left(f^{\prime} / f\right)(z)=e^{\alpha z} P(z) \psi(z)
$$

and therefore

$$
\left|\frac{f^{\prime}}{f}\left(r e^{i \theta}\right)\right|=\exp (\alpha r \cos \theta)\left|P\left(r e^{i \theta}\right)\right|\left|\psi\left(r e^{i \theta}\right)\right|
$$

where $\psi$ has only finitely many zeros and poles off the negative axis and satisfies (2.4) with $\omega<+\infty$. Let $z=r e^{i \theta} \in D_{2} \cup D_{3}$. Then $z$ is bounded away from the negative real axis and so by the Carathéodory estimate

$$
\left|\psi\left(r e^{i \theta}\right)\right| \geqslant k_{1}(\sin \varepsilon) r^{-1}=k r^{-1}
$$

where $k_{1}>0$ is a constant and $k=k_{1} \sin \varepsilon$. Letting $d=$ degree of $P,(5.3)$ and (5.4) give

$$
\left|\frac{f^{\prime}}{f}\left(r e^{i \theta}\right)\right| \geqslant k \exp \left(\alpha r \cos \left(\frac{\pi}{2}+\varepsilon\right)\right) r^{d-1} \quad\left(r \geqslant r_{0}\right) .
$$

Since $\alpha<0$ and $\cos (\pi / 2+\varepsilon)<0,(5.5)$ implies

$$
\left|\frac{f^{\prime}}{f}(z)\right| \rightarrow \infty \quad\left(|z| \rightarrow \infty, z \in D_{2} \cup D_{3}\right) \text {. }
$$

Let $z=r e^{i \theta} \in D_{1}$. Since $\psi$ has only finitely many positive zeros and poles, $\psi$ satisfies the conditions of Lemma 5; hence

$$
\left|\psi\left(r e^{i \theta}\right)\right|=O(1) \quad\left(r \rightarrow \infty, r e^{i \theta} \in D_{1}\right) .
$$


For $r e^{i \theta} \in D_{1},(5.3)$ and (5.7) give

$$
\left|\frac{f^{\prime}}{f}\left(r e^{i \theta}\right)\right| \leqslant C \exp \left(\alpha r \cos \left(\frac{\pi}{2}-\varepsilon\right)\right) r^{d} \quad\left(r \geqslant r_{1}, C>0 \text { constant }\right) .
$$

Then since $\alpha \cos (\pi / 2-\varepsilon)<0$,

$$
\frac{f^{\prime}}{f}(z) \rightarrow 0 \quad\left(|z| \rightarrow \infty, z \in D_{1}\right) .
$$

Notice that

$$
\frac{f^{\prime \prime}}{f^{\prime}}=\frac{\left(f^{\prime} / f\right)^{\prime}}{\left(f^{\prime} / f\right)}+\frac{f^{\prime}}{f}
$$

hence, by (5.2) we get

$$
\frac{f^{\prime \prime}}{f^{\prime}}(z)=\alpha+\frac{P^{\prime}}{P}(z)+\frac{\psi^{\prime}}{\psi}(z)+\frac{f^{\prime}}{f}(z) .
$$

Clearly

$$
\left|\left(\frac{P^{\prime}}{P}\right)(z)\right|=o(1) \quad(|z| \rightarrow \infty)
$$

and by Lemma 4

$$
\left|\left(\frac{\psi^{\prime}}{\psi}\right)(z)\right|=o(1) \quad\left(|z| \rightarrow \infty, z \in D=D_{1} \cup D_{2} \cup D_{3}\right) .
$$

Thus (5.6) and (5.9)-(5.12) imply

$$
\frac{f^{\prime \prime}}{f^{\prime}}(z) \rightarrow \alpha<0 \quad\left(|z| \rightarrow \infty, z \in D_{1}\right)
$$

and

$$
\left|\frac{f^{\prime \prime}}{f^{\prime}}(z)\right| \rightarrow \infty \quad\left(|z| \rightarrow \infty, z \in D_{2} \cup D_{3}\right) .
$$

Since $f^{\prime}(z) \neq 0$ for $|z|>R, z \in D,(5.13)$ and (5.14) imply $f^{\prime \prime}$ has only finitely many zeros in $D$.

Now if $F=1 / f$ note that by (5.2) and (3.29)

$$
\frac{F^{\prime \prime}}{F^{\prime}}=\alpha+\frac{P^{\prime}}{P}+\frac{\psi^{\prime}}{\psi}-\frac{f^{\prime}}{f} .
$$

Using an analysis identical to that above, it follows from (5.15) that $F^{\prime \prime}$ has only finitely many zeros in $D$. This completes the proof of Theorem 3 .

Proof of Corollary 3.1. Since $f$ and $f^{\prime}$ have finitely many zeros, (2.4) implies that $\psi$ is rational. Then for $\alpha<0$ (5.3) implies

$$
\left|\left(\frac{f^{\prime}}{f}\right)(z)\right| \rightarrow \infty \quad\left(|z| \rightarrow \infty, z \in E_{1}\right) .
$$

Further (5.10) and the rationality of $\psi$ imply

$$
\left|\left(\frac{f^{\prime \prime}}{f^{\prime}}\right)(z)\right| \rightarrow \infty \quad\left(|z| \rightarrow \infty, z \in E_{1}\right) .
$$


Similarly,

$$
\left(\frac{f^{\prime \prime}}{f^{\prime}}\right)(z) \rightarrow \alpha<0 \quad\left(|z| \rightarrow \infty, z \in D_{1}\right) .
$$

The result now follows from (5.15), (5.17) and (5.18). For $\alpha>0$, the argument is identical except that (5.17) holds in $D_{1}$ and (5.18) holds in $E_{1}$. The proof is complete.

Proof of Corollary 3.2. Since $f^{\prime}$ has finitely many zeros and at least one, $\psi_{1}$ is rational with

$$
\left|\psi_{1}(z)\right|=o(1) \quad(|z| \rightarrow \infty) .
$$

As in the proof of Corollary 3.1, if $\alpha<0$ (5.17) and (5.18) hold.

Fix $a \in \mathbf{C}$. If there exists an infinite sequence $\left\{\zeta_{n}\right\}_{n} \subseteq D_{1} \cup E_{1}$ such that $\phi_{1}\left(\zeta_{n}\right)=$ $a$, then by (5.19) and (1.10)

$$
\left|\frac{f^{\prime \prime}}{f^{\prime}}\left(\zeta_{n}\right)\right|=\left|\phi_{1}\left(\zeta_{n}\right)\right|\left|\psi_{1}\left(\zeta_{n}\right)\right|=a \cdot\left|\psi_{1}\left(\zeta_{n}\right)\right|=o(1) \quad(n \rightarrow \infty)
$$

contradicting (5.17) and (5.18). As in Corollary 3.1, an identical argument holds if $\alpha>0$. This completes the proof.

6. Proof of Theorem 4. Surely we may assume, by a simple translation if necessary, that zero is neither a zero nor pole of $\psi$. By Lemma $2, \psi^{\prime}$ has the form

$$
\psi^{\prime}(z)=\gamma+\sum_{k=\alpha}^{\omega} A_{k} \frac{1}{\left(z-a_{k}\right)^{2}}
$$

where $\gamma \geqslant 0, A_{k}>0$, and $a_{k}$ is real for all $k$. We are assuming $\psi^{\prime}(z)$ has only real zeros and (6.1) implies that $\psi^{\prime}(x)>0$ for all real $x \neq a_{k}$. Thus $\psi^{\prime}(z)$ has no zeros, and therefore

$$
g(z)=\left(\psi / \psi^{\prime}\right)(z)
$$

is entire with only real zeros. Also

$$
g^{\prime}(z)=1-\left[\psi(z) \psi^{\prime \prime}(z) /\left(\psi^{\prime}(z)\right)^{2}\right] .
$$

Since $\psi$ has only real zeros and we are assuming that $\psi^{\prime}$ and $\psi^{\prime \prime}$ have only real zeros, all the roots of

$$
g^{\prime}(z)=1
$$

are real. Then (6.2), (6.4) and Theorem D with $\beta=1$ and $\rho=0$ (since $g(z) \neq \infty$ in C) show that

$$
\lambda(g) \leqslant 1
$$

Also, since all the poles of $\psi$ are simple

$$
N\left(r, \frac{1}{g}\right)=N\left(r, \frac{\psi^{\prime}}{\psi}\right) \geqslant N(r, \psi) .
$$

Furthermore, since $\psi$ maps $\operatorname{Im} z>0$ to the upper half plane,

$$
m(r, \psi)=O(\log r) \quad(r \rightarrow \infty)
$$


and (6.5)-(6.7) give

$$
T(r, \psi) \leqslant O(\log r)+T(r, g) \quad(r \rightarrow \infty) .
$$

Thus

$$
\lambda(\psi) \leqslant 1 .
$$

It is easily seen that if in addition to the hypothesis of Theorem 4, it is also assumed that all but finitely many zeros and poles of $\psi$ lie on one ray $L$, where $L$ is either the negative or positive axis, then $\lambda(\psi) \leqslant \frac{1}{2}$.

To see this, we need only prove by (6.3) that all but finitely many zeros and poles of $\psi^{\prime \prime}$ lie on $L$, so that the analogue of $(6.4)$ holds. This is true because $\psi^{\prime \prime}$ has by hypothesis only real zeros and

$$
\psi^{\prime \prime \prime}(x)=\sum_{k=\alpha}^{\omega} \frac{6 A_{k}}{\left(x-a_{k}\right)^{4}} \quad(x=\operatorname{Re} z)
$$

has no real zeros. This means that $\psi^{\prime \prime}$ has at most one zero between consecutive poles of $\psi$ and at most one in each of $\left(-\infty, a_{\alpha}\right)$ and $\left(a_{\omega},+\infty\right)$. Hence all but finitely many zeros of $\psi^{\prime \prime}$ lie on $L$.

\section{REFERENCES}

1. A. Edrei, Meromorphic functions with three radially distributed values, Trans. Amer. Math. Soc. 78 (1955), 276-293.

2. W. K. Hayman, Meromorphic functions, Oxford Univ. Press, Oxford, 1964.

3. S. Hellerstein and J. Williamson, Derivatives of entire functions and a question of Pólya, Trans. Amer. Math. Soc. 227 (1977), 227-249.

4. (1977), 497-503.

Derivatives of entire functions and a question of Pólya. II, Trans. Amer. Math. Soc. 234 (2)

5 The zeros of the second derivative of the reciprocal of an entire function, Trans. Amer. Math. Soc. 263 (1981), 501-513.

6. B. Ja. Levin, Distribution of zeros of entire functions, GITTL, Moscow, 1956; English transl., Transl. Math. Monographs, vol. 5, Amer. Math. Soc., Providence, R. I., 1964.

7. B. Ja. Levin and I. V. Ostrovskii, On the dependence of the growth of an entire function on the distribution of the zeros of its derivatives (on a question of G. Pólya and A. Wiman), Sibirsk. Mat. Ž. 1 (1960), 427-455; English transl., Amer. Math. Soc. Transl. (2) 32 (1963), 323-357.

8. G. Pólya, Sur une question concernant les fonctions entières, C. R. Acad. Sci. Paris 158 (1914), $320-333$.

9. $392-400$ Bemerkung zur Theorie der ganzen Funktionen, Jber. Deutsch. Math.-Verein 24 (1915),

10. On the zeros of the derivatives of a function and its analytic character, Bull. Amer. Math. Soc. 49 (1943), 178-191.

11. J. Rossi, Two problems in entire function theory: Reciprocals of entire functions of infinite order and functions extremal for the $\cos \beta \lambda$ theorem, Doctoral dissertation, University of Hawaii, 1980.

12. W. Rudin, Real and complex analysis, 2nd ed., McGraw-Hill, New York, 1974.

Department of Mathematics, University of Hawail, Honolulu, Hawail 96822

Current address: Department of Mathematics, Purdue University, West Lafayette, Indiana 47906 